\title{
Challenge levels of everyday technologies as perceived over five years by older adults with mild cognitive impairment
}

\author{
Annicka Hedman, ${ }^{1}$ Anders Kottorp, ${ }^{1,2}$ Ove Almkvistt ${ }^{3,4}$ and Louise Nygård ${ }^{1}$ \\ ${ }^{1}$ Department of Neurobiology, Care Sciences and Society, Division of Occupational Therapy, Karolinska Institutet, Huddinge, Sweden \\ ${ }^{2}$ Faculty of Health and Society, Malmö University, Malmö, Sweden \\ ${ }^{3}$ Department of Neurobiology, Care Sciences and Society, Division of Clinical Geriatrics, Karolinska Institutet, Huddinge, Sweden \\ ${ }^{4}$ Department of Psychology, Stockholm University, Stockholm, Sweden
}

ABSTRACT

Background: In clinical practice, efficient and valid functional markers are needed to detect subtle cognitive and functional decline in mild cognitive impairment (MCI). This prospective study explored whether changes in perceived challenge of certain everyday technologies (ETs) can be used to detect signs of functional change in MCI.

Methods: Baseline and five-year data from 37 older adults (mean age 67.5 years) with MCI regarding their perceived ability to use ET were used to generate Rasch-based ET item measures reflecting the relative challenge of 46 ETs. Actual differential item functioning in relation to time was analyzed based on these item measures. Data collection took place in 2008-2014.

Results: Seven $(15 \%)$ of the ETs included were perceived to be significantly more challenging to use at year five compared to at baseline, while 39 ETs ( $85 \%$ ) were perceived to be equally challenging to use, despite the fact that the participants' perceived ability to use ET had decreased. Common characteristics among the ETs that became more challenging to use could not be identified. The dropout rate was $43 \%$, which limits the power of the study.

Conclusions: Changes in the perceived challenge of ETs seem to capture functional change in persons with cognitive decline. Both easier and more challenging ETs typically used at home and in society need to be addressed to capture this functional change because significant changes occurred among ETs of all challenge levels and within all types of ETs.

Key words: longitudinal studies, dementia, activities of daily living (ADLs)

\section{Introduction}

Mild cognitive impairment (MCI) is a heterogeneous clinical syndrome that lies between the cognitive functioning in normal aging and the early stage of dementia (Petersen et al., 2014). Core clinical criteria in former and current major definitions of MCI include roughly preserved independence in functional abilities despite some objectively verified cognitive impairment and selfor informant-reported cognitive symptoms, thus not dementia (Petersen, 2004; Winblad et al., 2004; American Psychiatric Association, 2013). This description overlaps with criteria intended

Correspondence should be addressed to: Annicka Hedman, Department of Neurobiology, Care Sciences and Society (NVS), Division of Occupational Therapy, Karolinska Institutet, Fack 23 200, SE-141 83 Huddinge, Sweden. Phone: +46 (8) 524837 52. Email: annicka.hedman@ki.se. Received 27 Nov 2017; revision requested 28 Feb 2018; revised version received 1 Feb 2018; accepted 13 Feb 2018. First published online 4 April 2018. to describe preclinical Alzheimer's disease (AD) (Albert et al., 2011). To date, no effective treatment of MCI is available (Kane et al., 2017). Nevertheless, early detection of morbid cognitive and functional decline remains important; in the current situation in order to enable adequate and targeted support to handle the consequences of such declines in everyday life, and in the future to identify the optimal therapeutic window (Belleville et al., 2014). In clinical practice, efficient and valid functional markers are needed that capture the subtle cognitive and functional decline in MCI. Impaired ability to manage finances and medications (Arrighi et al., 2013) and withdrawal from participating in outings and other leisure activities (Arrighi et al., 2013; Hedman et al., 2016) have been suggested as early functional signs of cognitive change. In addition, changes in the ability to use everyday technologies (ETs), i.e. the wide range of technical objects and services that are 
commonly present and used in our everyday lives at home and in the community, have proven to be one such potential marker (Malinowsky et al., 2010; Nygård et al., 2012; Malinowsky et al., 2017). ET use can, for example, be studied by assessing the ability of individuals to use specific ETs or by focusing on the challenge level of specific ETs as perceived by users. This prospective five-year study explored whether the challenge level of certain ETs is more sensitive and well suited to detecting signs of functional change in MCI.

Cognitive ability has been shown to affect how many types of ETs people use (Czaja et al., 2006) and how efficient this use is (Slegers et al., 2009). On a group level, both perceived (Nygård et al., 2012) and observed (Malinowsky et al., 2010) ability to use ETs are significantly lower in persons with MCI compared to in persons with no known cognitive impairment, and even lower in persons with dementia. Ability in ET use as well as amount of ETs used have shown a potential to differentiate groups with different needs of assistance among persons with cognitive impairment (Ryd et al., 2016). Furthermore, a declining pattern regarding the ability to use ETs and activity involvement during the years following the detection of MCI might be predictive of future dementia (Hedman et al., 2017a).

The cognitive decline in MCI includes impaired memory related to recollective/associative abilities and short-term retention, impaired executive functions in new or demanding tasks, and reduced attentional control (Belleville et al., 2014), which together with visuo-spatial function all exemplify cognitive abilities needed when using ETs such as coffee makers, cell phones, and cash machines (ATMs). Performance skills, i.e. observable actions when performing everyday activities, often involved when managing ETs and known to be, especially challenging are choosing the correct button or command, identifying the services or functions of the ET, and performing actions in a logical sequence (Malinowsky et al., 2011). We also know that certain ET characteristics make their use more challenging for persons with and without cognitive impairments. ETs demanding a high frequency of performance skill actions, requiring use of more difficult performance skills, failing to provide feedback related to a variety of sensory functions (e.g. visual, auditory, and tactile), having complex design, and typically being infrequently used, pose higher levels of challenge on the users (Patomella et al., 2011, 2013). Thus, complexity in process might be the common denominator challenging both cognition and ET use. Cross-sectional research has suggested that
Table 1. Participant characteristics at inclusion

$(n=37)$

\begin{tabular}{ll}
\hline VARIABLE & VALUE \\
Sex, $n(\%)$ & \\
$\quad$ Female & $18(49)$ \\
$\quad$ Male & $19(51)$ \\
Age, years & \\
$\quad$ Mean (SD) & $67.4(7.5)$ \\
$\quad$ Min-max & $56-82$ \\
Living condition, $n(\%)$ & \\
$\quad$ Cohabiting & $28(76)$ \\
$\quad$ Living alone & $9(24)$ \\
Education, years & \\
$\quad$ Mean (SD) & $12.6(3.3)$ \\
$\quad$ Min-max & $6-20$ \\
ETUQ person measure & \\
$\quad$ Mean (SD) & $54.6(2.8)$ \\
$\quad$ Min-max & $48.5-60.6$ \\
MSE & \\
$\quad$ Median (IQR) & $28(2)$ \\
$\quad$ Min-max & $19-30$ \\
\hline
\end{tabular}

Note: Mean and SD are presented for normally distributed data, while median and IQR are given for skewed data. ETUQ:

Everyday Technology Use Questionnaire, where higher person measure indicates higher perceived ability in ET use (Nygård et al., 2012). MMSE: Mini-Mental State Examination (0-30), where higher score indicates better cognitive status (Folstein et al., 1975).

domestic ETs such as coffee makers, stoves, and microwave ovens are generally perceived and observed to be easier to use, while information and communication technologies (ICT) like cell phones and computers are more challenging (Patomella et al., 2011; Malinowsky et al., 2013). However, longitudinal studies examining how the perceived challenge of specific ETs develops in persons with cognitive decline are lacking. Identifying ETs whose challenge level has the potential to detect functional change might be highly interesting for ensuring targeted support at an early stage. Therefore, we examined how older adults perceived the challenge levels of commonly used ETs at the time of MCI detection and five years later, with the aim to investigate whether the use of certain ETs is more sensitive to cognitive decline.

\section{Methods}

\section{Sample}

Participants were older adults with MCI diagnosed and recruited at a specialized outpatient memory clinic in Stockholm between April 2008 and May 2009 (Table 1). The inclusion criteria were: (a) fulfilled criteria for MCI as proposed by (Petersen, 2004 ), i.e. self-rated and clinically verified cognitive decline, no dementia, and essentially intact basic 


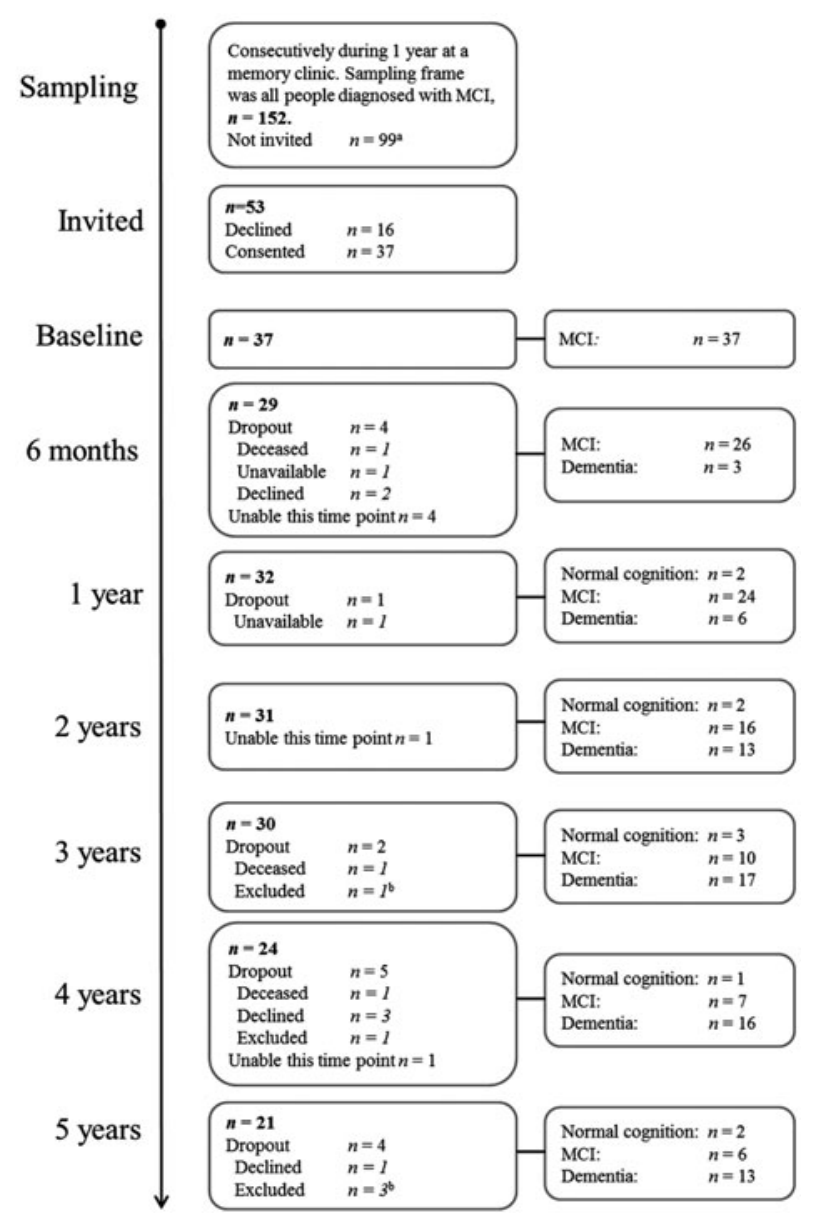

Figure 1. Overview of sampling, data available and missing, and diagnostic information at the seven follow-up occasions.

Notes: ${ }^{\text {aThose }}$ who did not meet inclusion criteria $(n=71)$ were not invited. Other reasons $(n=28)$ for not being invited included, for example, social problems and living too far away from the clinic for practical data collection.

${ }^{\mathrm{b}}$ Exclusion based on ethical considerations.

and instrumental activities of daily living; (b) at least 55 years old; (c) being a user of ETs; (d) the ability to take part in data collection in Swedish; (e) no cognitive comorbidities; and (f) no severe problems with hearing or vision that could not be compensated for. The Regional Ethics Committee in Stockholm approved the study, and informed consent was obtained from all participants. Figure 1 provides an overview of the sampling and of the data that were available and missing at the seven follow-up occasions over the five-year period. As the inclusion ended 2009, the final 5-year-follow-ups took place in 2014.

\section{Data collection}

In the present study, we used baseline and fiveyear data because a previous study using the same sample (Hedman et al., 2017b) had shown a significant decrease in perceived ability to use
Table 2. Description of the scale steps in the ETUQ

SCORE DESCRIPTION
$\begin{aligned} & \text { The ET is used with no hesitation or } \\ & \text { difficulty at all } \\ & \text { The ET is used with minor hesitation or } \\ & \text { difficulty } \\ & \text { The ET is used with frequent/major } \\ & \text { difficulties } \\ & \text { The ET is sometimes/partly used together } \\ & \text { with another person } \\ & \text { The ET is only used together with another } \\ & \text { person } \\ & \text { The ET is not used anymore, or has not come } \\ & \text { into use, even if it is available and relevant } \\ & \text { for the person }\end{aligned}$
No score $\quad$ Non-relevant

ETs only when comparing year five and baseline, and not between consecutive time points. The first author and five research assistants conducted the structured face-to-face interviews, most often in the homes of the participants. Those who so wished were accompanied by a significant other for support, but we based the scoring on the participants' answers. To capture longitudinal changes in the perceived level of challenge of a range of ETs of varying difficulty, the Everyday Technology Use Questionnaire (ETUQ) was used (Rosenberg et al., 2009). The ETUQ includes 92 ETs commonly used by older adults inside and outside the home. Each ET perceived as relevant by the person is scored on a 6-step scale, rating the person's perceived ability to use it (Table 2). In the ETUQ, a relevant ET is defined as an ET that the person has access to and has used in the past, currently is using, or intends to start using. In this study, both person measures and item measures were used (further explained in the data analysis section). The ETUQ has shown acceptable psychometric properties (unidimensionality, rating scale validity, and person response validity) in studies including older adults with cognitive impairments (Rosenberg et al., 2009; Nygård et al., 2012). Additionally, cognitive function was assessed using the Mini-Mental State Examination (MMSE) (Folstein et al., 1975), and we obtained diagnostic information from medical files at the memory clinic.

\section{Data analysis}

We used a Rasch model to transform the ordinal ETUQ scores into relative linear measures expressed in log odds probability units (logits) (Bond and Fox, 2007). This is a suitable approach when analyzing ETUQ data because Rasch models 
can handle the typical scenario that not all ETUQ items are scored as relevant by all participants. That is, the response patterns of all the participants are used by the model to generate individual person measures, reflecting the perceived ability in ET use, as well as item measures, reflecting the relative challenge of each ET. These measures are displayed in two different hierarchy outputs, one placing the participants on a continuum from lower to higher perceived ability to use ETs, and another one placing the ETs on a continuum from being perceived as less to more challenging to use. A higher person measure indicates higher perceived ability in ET use for a person, while a higher item measure indicates a higher level of perceived challenge for an ET. In this study, special focus was placed on the item measures because our interest was to explore whether the challenge level of certain ETs was more sensitive to cognitive decline. However, we ensured stability and validity of the person measures by securing proper rating scale functioning according to standard procedures described earlier (Nygård et al., 2012).

For an ET to be included in the analysis, at least ten participants should have scored it as relevant both at baseline and at year five. This was true for 46 of the 92 items in the ETUQ $(50 \%)$. In order to detect whether specific ETs presented more, or less, challenge over five-years' time than expected based upon the Rasch model, we examined differential item functioning (DIF) in relation to time. To adjust for changes in the mean person measure of the sample, actual DIF (Petersson et al., 2008) between baseline and year five was calculated for each ET through the use of standardized $z$-comparisons calculated on the individual item measures from both of these time points and their individual standard errors. We used a $z$-score difference of $\geq \pm 1.96$ as the criterion for significant actual DIF, and an item measure of 50.0 logits was used as a cut-off to classify the items as "relatively more easy" or "relatively more challenging" to use at baseline. An item measure of 50.0 logits represents an item where a person with a similar person measure (i.e. 50.0 logits) has an equal chance of scoring a 3 (The ET is sometimes/partly used together with another person) or a 4 (The ET is used with frequent/major difficulties), i.e. the cut-off between using the ET with or without another person. To explore potential patterns in the types of ETs that presented significant actual DIF, the analyzed ETs were also classified into the seven topic areas in which all ETs are organized in the latest ETUQ version (Nygård et al., 2016) - home maintenance, information/communication, self-care, maintenance/repair, accessibility, economy/purchasing, and travel.

\section{Results}

At year five, 21 of the 37 included participants contributed with data, which gives a dropout rate of $43 \%$. An independent-sample $t$-test revealed no significant baseline differences regarding age, MMSE, or ETUQ person measure between those who contributed with data at year five and those who had dropped out, and a $\chi^{2}$ test indicated no significant relation between gender and attrition. The mean perceived ability to use ETs was 54.6 (SD 2.8) logits at baseline and 52.2 (SD 3.1) logits at year five. These person measures were used when adjusting the standardized $z$-score differences examining the actual DIF of the included ETs.

In Table 3, ET item measures of challenge at baseline and year five, standardized $z$-score differences, and item types of the 46 included ETs are presented. In total, seven (15\%) of the analyzed ETs had positive $z$-score differences that were outside the \pm 1.96 interval, i.e. they showed significant actual DIF over time. The positive scores indicate that these ETs were perceived to become more challenging to use over time. For $39(85 \%)$ of the ETs, no significant actual DIF was found, indicating overall significantly stable perceived challenge level over time for these ETs despite overall decreasing ETUQ person measures in the sample. A total of 30 of the 46 analyzed ETs $(65 \%)$ were classified as "relatively more easy" to use at baseline, while $16(35 \%)$ were classified as "relatively more challenging" to use. At year five, 33 ETs $(72 \%)$ were classified as "relatively more easy", while $13(28 \%)$ were classified as "relatively more challenging." Among the 30 ETs classified as "relatively more easy" to use at baseline, five (17\%) - washing machine, TV with remote control, handheld mixer, internet interaction, and cash machine $(A T M)$ - showed significant actual DIF, indicating that these became more challenging to use over time. Among the $16 \mathrm{ET}$ s classified as "relatively more challenging" to use at baseline, two (13\%) stereo/CD player and digital camera - showed significant actual DIF, which reflects that they became even more challenging to use over time.

As can be seen in Table 3, ET item types within the ETUQ topic areas of self-care, maintenance/repair, and travel are not represented in the analysis due to a small number of scorings.

\section{Discussion}

Our findings show that $15 \%$ of the analyzed ETs were significantly more challenging to use at year five compared to at baseline. Based on chance, we would have expected that just over two of the 
Table 3. Item measures, standardized $z$-score differences examining actual DIF, and ET types for the included ETs, here ordered according to their challenge level at baseline with a cut-off for "relatively more easy" versus "relatively more difficult" to use at 50.0 logits

\begin{tabular}{|c|c|c|c|c|}
\hline & & & Z-SCORE & \\
\hline & ITEM & & DIFFER- & \\
\hline & MEASURE IN & ITEM & ENCE & \\
\hline & LOGITS AT & MEASURE IN & BETWEEN & \\
\hline ET $(N=46)$ & $\begin{array}{l}\text { BASELINE } \\
(S E)\end{array}$ & $\begin{array}{l}\text { LOGITS AT } \\
\text { YEAR } 5 \text { (SE) }\end{array}$ & $\begin{array}{l}\text { BASELINE } \\
\text { AND YEAR } 5\end{array}$ & ET TYPE \\
\hline Elevator & $29.36(9.88)$ & $20.55(18.27)$ & -0.31 & Accessibility \\
\hline Vacuum cleaner & $29.60(9.87)$ & $46.07(1.70)$ & 1.88 & Home maintenance \\
\hline $\begin{array}{l}\text { Answering machine: leave a } \\
\text { message when calling }\end{array}$ & $30.11(9.87)$ & $45.16(2.33)$ & 1.72 & Information/Comm. \\
\hline Dishwasher & $30.57(9.82)$ & $46.37(1.79)$ & 1.82 & Home maintenance \\
\hline Washing machine & $37.45(6.36)$ & $48.50(1.38)$ & 2.07 & Home maintenance \\
\hline Coffee maker/coffee machine & $44.89(2.31)$ & $47.56(1.66)$ & 1.78 & Home maintenance \\
\hline $\begin{array}{l}\text { Ticket-operated queuing } \\
\text { system }\end{array}$ & $45.19(2.16)$ & $44.49(2.35)$ & 0.54 & Accessibility \\
\hline Stove & $46.22(1.80)$ & $48.14(1.36)$ & 1.92 & Home maintenance \\
\hline $\begin{array}{l}\text { Flushing mechanism in public } \\
\text { restroom }\end{array}$ & $46.37(2.10)$ & $45.82(2.05)$ & 0.64 & Accessibility \\
\hline Iron & $46.43(1.88)$ & $47.44(1.49)$ & 1.43 & Home maintenance \\
\hline Electric kettle & $46.94(1.85)$ & $36.67(8.65)$ & -0.89 & Home maintenance \\
\hline $\begin{array}{l}\text { Automated faucet/dryer in } \\
\text { public restroom }\end{array}$ & $47.06(1.86)$ & $44.11(2.53)$ & -0.17 & Accessibility \\
\hline Radio & $47.08(1.80)$ & $46.99(1.62)$ & 0.96 & Information/Comm. \\
\hline $\begin{array}{l}\text { Push-button to get off bus or } \\
\text { open bus door }\end{array}$ & $47.11(1.58)$ & $47.11(1.84)$ & 0.99 & Accessibility \\
\hline TV with remote control & $47.28(1.53)$ & $49.14(1.25)$ & 2.16 & Information/Comm. \\
\hline Hand-held mixer & $47.69(1.53)$ & $51.14(1.42)$ & 2.80 & Home maintenance \\
\hline Internet interaction & $47.74(1.69)$ & $51.51(1.40)$ & 2.81 & Information/Comm. \\
\hline Calculator & $48.04(1.44)$ & $48.03(1.79)$ & 1.05 & Economy/Purchasing \\
\hline Microwave oven & $48.12(1.40)$ & $48.31(1.34)$ & 1.34 & Home maintenance \\
\hline Digital thermometer & $48.16(1.58)$ & $44.01(2.50)$ & -0.59 & Home maintenance \\
\hline Egg timer & $48.29(1.48)$ & $43.17(3.20)$ & -0.77 & Home maintenance \\
\hline Dryer & $48.61(1.45)$ & $48.99(1.65)$ & 1.27 & Home maintenance \\
\hline Alarm clock/clock radio & $48.81(1.27)$ & $47.91(1.54)$ & 0.76 & Home maintenance \\
\hline Toaster & $49.19(1.23)$ & $44.73(2.79)$ & -0.67 & Home maintenance \\
\hline Push-button telephone & $49.19(1.28)$ & $42.53(3.68)$ & -1.09 & Information/Comm. \\
\hline Cell phone: charger & $49.35(1.21)$ & $46.98(1.56)$ & 0.02 & Information/Comm. \\
\hline Portable telephone, cordless & $49.50(1.19)$ & $47.64(1.70)$ & 0.26 & Information/Comm. \\
\hline Cash machine, ATM & $49.53(1.21)$ & $51.33(1.11)$ & 2.56 & Economy/Purchasing \\
\hline Loyalty card & $49.67(1.24)$ & $49.32(1.40)$ & 1.10 & Economy/Purchasing \\
\hline Internet information search & $49.90(1.39)$ & $50.33(1.31)$ & 1.49 & Information/Comm. \\
\hline Cell phone: other & $50.01(1.54)$ & $47.40(2.14)$ & -0.08 & Information/Comm. \\
\hline Cell phone: make call & $50.14(1.10)$ & $47.66(1.45)$ & -0.04 & Information/Comm. \\
\hline Cell phone: receive call & $50.26(1.10)$ & $46.19(1.71)$ & -0.82 & Information/Comm. \\
\hline $\begin{array}{l}\text { Credit/debit card with PIN } \\
\text { code }\end{array}$ & $50.46(1.10)$ & $49.86(1.27)$ & 1.08 & Economy/Purchasing \\
\hline Door code & $50.69(1.06)$ & $48.35(1.46)$ & 0.04 & Accessibility \\
\hline Smoke detector & $50.73(1.17)$ & $50.51(1.29)$ & 1.25 & Home maintenance \\
\hline $\begin{array}{l}\text { Automated telephone-based } \\
\text { functions (e.g. number } \\
\text { selector, voice control) }\end{array}$ & $50.97(1.04)$ & $47.23(1.69)$ & -0.67 & Information/Comm. \\
\hline Stereo/CD player & $51.03(1.04)$ & $52.29(1.30)$ & 2.20 & Information/Comm. \\
\hline Entry phone & $51.38(1.13)$ & $48.14(1.61)$ & -0.42 & Accessibility \\
\hline $\begin{array}{l}\text { Receiver of digital TV (e.g. } \\
\text { converter box) }\end{array}$ & $51.45(1.30)$ & $52.87(1.69)$ & 1.80 & Information/Comm. \\
\hline
\end{tabular}


Table 3. Continued

\begin{tabular}{|c|c|c|c|c|}
\hline & & & $Z-S C O R E$ & \\
\hline & ITEM & & DIFFER- & \\
\hline & MEASURE IN & ITEM & ENCE & \\
\hline & LOGITS AT & MEASURE IN & BETWEEN & \\
\hline ET $(N=46)$ & $\begin{array}{l}\text { BASELINE } \\
(S E)\end{array}$ & $\begin{array}{l}\text { LOGITS AT } \\
\text { YEAR } 5(\mathrm{SE})\end{array}$ & $\begin{array}{l}\text { BASELINE } \\
\text { AND YEAR } 5\end{array}$ & ET TYPE \\
\hline Camera, digital & $51.52(1.10)$ & $54.87(1.64)$ & 2.92 & Information/Comm. \\
\hline Internet banking & $51.52(1.29)$ & $50.18(1.73)$ & 0.50 & Economy/Purchasing \\
\hline Remote control: other & $52.21(1.15)$ & $53.21(1.57)$ & 1.76 & Accessibility \\
\hline Cell phone: text message & $52.64(1.13)$ & $50.17(1.58)$ & -0.03 & Information/Comm. \\
\hline DVD & $53.01(0.96)$ & $54.01(1.50)$ & 1.91 & Information/Comm. \\
\hline Video & $55.45(0.90)$ & $56.35(1.90)$ & 1.57 & Information/Comm. \\
\hline
\end{tabular}

Note: Positive $z$-score difference reflects increasing difficulty of the ET item over time. Negative $z$-score difference reflects decreasing difficulty of the ET item over time. ETs in bold print reach or exceed the $\geq \pm 1.96$ cut-off for significant actual DIF.

46 ETs (5\%) would demonstrate significant DIF. The findings exceeded this number. However, the majority of the ETs (85\%) were perceived to be within a similar challenge level five years after inclusion, despite the fact that the participants' perceived overall ability to use ETs had decreased and the fact that the diagnostic composition in the sample had changed from $100 \%$ MCI to $62 \%$ dementia. These findings deserve some reflection.

It is difficult to distinguish common characteristics among the seven ETs that showed significant DIF in this study, in relation to the ETs that did not demonstrate DIF over time. Some of the seven ETs that became significantly more challenging to use could be linked to activities known to reveal early functional signs of cognitive change. For example, perceiving the cash machine (ATM) as more challenging to use is in line with earlier findings of impaired ability to manage finances (Arrighi et al., 2013), and the increased challenge level in using ETs like TV with remote control, hand-held mixer, internet interaction, stereo/CD player, and digital camera might be linked to the known early withdrawal from leisure activities in cognitive decline (Arrighi et al., 2013; Hedman et al., 2016). However, several similar ETs typically used in similar activities were also found among the ETs with stable challenge level, for example, internet banking, receiver of digital $T V$, and internet information search. Similarly, explanations linked to the complexity of the activities where the ETs were used, or the complexity of the actions involved in the ET use (Malinowsky et al., 2011), were not applicable.

The generated hierarchy displaying the ETs on a continuum from less to more challenging to use (Table 3) reaffirms earlier knowledge of domestic ETs as generally being easier to use (Patomella et al., 2011; Malinowsky et al., 2013) because all but one ET within home maintenance were found among the "relatively more easy" ETs. Somewhat unexpectedly, a larger proportion of ETs classified as "relatively more easy" to use at baseline became significantly more challenging to use at year five $(17 \%)$ compared to the proportion of ETs with significant DIF that at baseline were classified as "relatively more challenging" (13\%). At baseline, the participants likely managed to use the washing machine, TV with remote control, handheld mixer, internet interaction, and cash machine (ATM) without the support of another person, albeit with varying degrees of difficulty. By year five, however, several of these ETs had passed over to the "relatively more challenging" side of the 50.0 logit cut-off, thus becoming more likely for the persons to use with another person's support. This finding has at least two clinical implications. First, in order to detect possible functional decline it might be appropriate to ask persons with MCI whether there are ETs that they previously could manage by themselves that now require support from another person. Second, it is relevant to also attend to less challenging ETs within the area of home maintenance and not focus only on high-tech ETs within the areas of information/communication and economy/purchasing because home maintenance involved ETs with significant DIF.

Our finding of a predominantly stable challenge level of ETs over time corresponds in part with a previous study comparing the challenge level of ETs at two time periods three to five years apart in two different samples, including persons with MCI, persons with dementia, and controls (Malinowsky et al., 2015). Also, in that study, stable challenge levels were most common with $85 \%$ stable ETs. However, contrary to the findings in our study, all but one of the ETs showing a significant change in the level of challenge were perceived as easier to use at the later time period 
in the previous study. This difference might be due to the fact that in the previous study there were different individuals included in the different time periods and the fact that the study also included older adults without cognitive impairments. The direction of the findings in the present study indicates that our underlying assumption might be correct; the challenge levels of certain ETs seem to be more sensitive to cognitive change and might thereby be a useful indicator of functional change in MCI. However, we are reluctant to highlight the specific ETs that showed significant DIF in this study as being, especially sensitive to cognitive decline because no clear patterns regarding their characteristics in comparison to the stable items could be found. We instead propose a comprehensive approach when evaluating the challenge levels of ETs over time in older adults with MCI and dementia, including both easier and more challenging ETs and including domestic ETs as well as ICT.

Given the lack of earlier longitudinal research that explores ET use on the item level in older adults with cognitive decline, this DIF study was undertaken despite the risk of being underpowered. The findings should therefore be interpreted with caution. As a consequence of the large attrition rate of $43 \%$, the sets of persons generating the item measures differ between baseline and year five. However, the dropout analysis shows that the attrition did not differ at baseline from those who contributed with data at year five regarding MMSE, ability to use ETs, gender, or age, which suggests that the generated item calibrations at year five would have been similar without the attrition. Finally, it is important to remember that changes in challenge levels of specific ETs may occur for reasons other than functional change in the participants. For example, updating or replacement of technologies can also contribute to changing challenge levels. Although people with MCI generally are reluctant to replacing ETs with new ones (Nygård, 2008), it is possible that some ETs used by the participants were altered or replaced during the five years. For instance, new features could have made a cell phone easier or more challenging to use. Thus, ET use needs to be interpreted from several perspectives, not only based on the person's characteristics and diagnosis.

In conclusion, the findings of this study suggest that change of perceived challenge of ETs may indicate functional change in persons with cognitive decline, even if alternative explanations cannot be ruled out. Both easier and more challenging ETs typically used at home and in society need to be addressed to capture this functional change because significant changes may occur among ETs of all challenge levels and within all types of ETs in older people with MCI.

\section{Conflict of interest}

None.

\section{Description of authors' roles}

All authors formulated the research question in consensus. Hedman took part in data collection, performed the statistical analysis, and wrote the paper. Kottorp was responsible for the statistical design of the study, gave advice during the statistical analysis, and provided input to the draft. Almkvist gave advice regarding the statistical analysis and provided input to the draft. Nygård planned the 5-year study, supervised the data collection, and assisted in writing the paper.

\section{Acknowledgments}

We thank the participants for sharing their experiences of ET use with us and the occupational therapists who helped us with recruitment and data collection. The following funders made the project possible: the Swedish Council for Health, Working Life and Welfare (FORTE); the Swedish Research Council (VR); the Strategic Research Programme in Care Sciences at the Karolinska Institutet; and the regional agreement on medical training and clinical research (ALF) between Stockholm County Council and the Karolinska Institutet.

\section{References}

Albert, M. S. et al. (2011). The diagnosis of mild cognitive impairment due to Alzheimer's disease: recommendations from the national institute on Aging-Alzheimer's association workgroups on diagnostic guidelines for Alzheimer's disease. Alzheimers $\&$ Dementia, 7, 270-279.

American Psychiatric Association (2013). Diagnostic and Statistical Manual of Mental Disorders: DSM-5. Arlington, VA: American Psychiatric Association.

Arrighi, H. M., Gélinas, I., Mclaughlin, T. P., Buchanan, J. and Gauthier, S. (2013). Longitudinal changes in functional disability in Alzheimer's disease patients. International Psychogeriatrics, 25, 929-937.

Belleville, S., Fouquet, C., Duchesne, S., Collins, D. L., Hudin, C. and The Cima-Q Group (2014). Detecting early preclinical Alzheimer's disease via cognition, neuropsychiatry, and neuroimaging: qualitative review and recommendations for testing. Fournal of Alzheimer's Disease, 42, S375-S382. 
Bond, T. G. and Fox, C. M. (eds.) (2007). Applying the Rasch Model: Fundamental Measurement in the Human Sciences. Mahwah, N J: Lawrence Erlbaum Associates.

Czaja, S. J. et al. (2006). Factors predicting the use of technology: findings from the Center for Research and Education on Aging and Technology Enhancement (CREATE). Psychology and Aging, 21, 333-352.

Folstein, M. F., Folstein, S. E. and Mchugh, P. R. (1975). "Mini-mental state". A practical method for grading the cognitive state of patients for the clinician. Fournal of Psychiatric Research, 12, 189-198.

Hedman, A., Kottorp, A. and Nygård, L. (2017a). Patterns of everyday technology use and activity involvement in mild cognitive impairment: a five-year follow-up study. Aging $\mathcal{E}$ Mental Health. Epub ahead of print. doi:10.1080/13607863.2017.1297361.

Hedman, A., Nygard, L. and Kottorp, A. (2017b). Everyday technology use related to activity involvement among people in cognitive decline. American fournal of Occupational Therapy September/October, 71, 71051900401-71051900408.

Hedman, A., Nygård, L., Malinowsky, C., Almkvist, 0. and Kottorp, A. (2016). Changing everyday activities and technology use in mild cognitive impairment. British Fournal of Occupational Therapy, 79, 111-119.

Kane, R. L. et al. (2017). Interventions To Prevent Age-Related Cognitive Decline, Mild Cognitive Impairment, and Clinical Alzheimer's-Type Dementia. Comparative Effectiveness Review No. 188. (Prepared by the Minnesota Evidence-based Practice Center under Contract No. 290-2015-00008-I.) Rockville, MD: Agency for Healthcare Research and Quality. AHRQ Publication No. 17-EHC008-EF. Available at: http://www. effectivehealthcare.ahrq.gov/reports/final.cfm; last accessed 19 March 2018.

Malinowsky, C., Almkvist, O., Kottorp, A. and Nygård, L. (2010). Ability to manage everyday technology: a comparison of persons with dementia or mild cognitive impairment and older adults without cognitive impairment. Disability and Rehabilitation: Assistive Technology, 5, 462-469.

Malinowsky, C. et al. (2017). Differences in the use of everyday technology among persons with MCI, SCI and older adults without known cognitive impairment. International Psychogeriatrics, 29, 1193-1200.

Malinowsky, C., Kottorp, A. and Nygård, L. (2013). Everyday technologies' levels of difficulty when used by older adults with and without cognitive impairment Comparison of self-perceived versus observed difficulty estimates. Technology and Disability, 25, 167-176.

Malinowsky, C., Kottorp, A., Patomella, A., Rosenberg, L. and Nygård, L. (2015). Changes in the technological landscape over time: relevance and difficulty levels of everyday technologies as perceived by older adults with and without cognitive impairment. Technology and Disability, 27, 91-101.
Malinowsky, C., Nygård, L. and Kottorp, A. (2011). Psychometric evaluation of a new assessment of the ability to manage technology in everyday life. Scandinavian Fournal of Occupational Therapy, 18, 26-35.

Nygård, L. (2008). The meaning of everyday technology as experienced by people with dementia who live alone. Dementia, 7, 481-502.

Nygård, L., Pantzar, M., Uppgard, B. and Kottorp, A. (2012). Detection of activity limitations in older adults with MCI or Alzheimer's disease through evaluation of perceived difficulty in use of everyday technology: a replication study. Aging $\mathcal{E}$ Mental Health, 16, 361371.

Nygård, L., Rosenberg, L. and Kottorp, A. (2016). Users Manual: Everyday Technology Use Questionnaire (ETUQ). Karolinska Institutet, Division of Occupational Therapy. Unpublished manuscript.

Patomella, A. H., Kottorp, A., Malinowsky, C. and Nygård, L. (2011). Factors that impact the level of difficulty of everyday technology in a sample of older adults with and without cognitive impairment. Technology and Disability, 23, 243-250.

Patomella, A. H., Kottorp, A. and Nygård, L. (2013). Design and management features of everyday technology that challenge older adults. The British fournal of Occupational Therapy, 76, 390-398.

Petersen, R. C. (2004). Mild cognitive impairment as a diagnostic entity. Fournal of Internal Medicine, 256, 183-194.

Petersen, R. C., Caracciolo, B., Brayne, C., Gauthier, S., Jelic, V. and Fratiglioni, L. (2014). Mild cognitive impairment: a concept in evolution. Fournal of Internal Medicine, 275, 214-228.

Petersson, I., Lilja, M., Hammel, J. and Kottorp, A. (2008). Impact of home modification services on ability in everyday life for people aging with disabilities. Fournal of Rehabilitation Medicine, 40, 253-260.

Rosenberg, L., Nygård, L. and Kottorp, A. (2009). Everyday technology use questionnaire: psychometric evaluation of a new assessment of competence in technology use. OTFR, 29, 52-62.

Ryd, C., Nygård, L., Malinowsky, C., Öhman, A. and Kottorp, A. (2016). Can the everyday technology use questionnaire predict overall functional level among older adults with mild cognitive impairment or mild-stage Alzheimer's disease? - A pilot study. Scandinavian fournal of Caring Sciences, 31, 201-209.

Slegers, K., Van Boxtel, M. P. J. and Jolles, J. (2009). The efficiency of using everyday technological devices by older adults: the role of cognitive functions. Ageing $\mathcal{E}$ Society, 29, 309-325.

Winblad, B. et al. (2004). Mild cognitive impairment-beyond controversies, towards a consensus: report of the International Working Group on Mild Cognitive Impairment. Fournal of Internal Medicine, 256, 240-246. 